\title{
A system for data processing in haematology
}

\author{
I. CAVIll, C. RiCKetTS, T. MOULDing, A. JACOBS, AND M. PAGE
}

From the Department of Haematology, University Hospital of Wales, Heath Park, Cardiff, and the Welsh Hospital Board Computer Centre

SYNOPSIS The data processing problems which arise in the provision of a modern haematology service are discussed in the light of available resources, and the basic requirements of any automated data processing system are defined. The differences between the needs of haematology and those of other pathology specialities are emphasized. A strategy for the development of a simple system which covers data capture, analysis, and retrieval is described. Separation of the short-and longterm functions of the system provides a relatively simple, inexpensive solution which can be modulated to meet the particular circumstances of each laboratory.

The advent of computers in medicine is often regarded with a foreboding that appears justified by the prospect of complex, all-embracing schemes which may appear to relegate the doctor and his patient to a position of subservience. Moreover, the success of such schemes depends on the simultaneous solution of a wide range of problems under conditions which may be inadequately defined.

We have recently considered the possibility of a scheme for integrated data processing in all branches of pathology. Our experience in Cardiff has suggested that this could only be achieved by devoting a disproportionate part of the potential resources of the division to this aim. It would require a large capital allocation for what would necessarily be something of an experimental project and would require a relatively large number of highly specialized staff. Those concerned with the individual specialities within pathology therefore decided on a period of independent development while bearing in mind the aim of eventual union. This simplified approach has allowed each specialty to develop on relatively limited budgets and within the capabilities of its existing staff structure. We present our own experience as a case history illustrating not only how the problems of haematology can be tackled but an approach that may be useful in other areas.

\section{The Problem}

During the last decade there has been an explosive increase in the work of haematology laboratories

Received for publication 23 January 1974. and only automation of the commonest procedures has allowed haematologists to meet this rising demand. Data production has now risen to such an extent that data processing is becoming an important factor limiting the effectiveness of the laboratory. The introduction of automated data processing techniques to facilitate laboratory work flow and reporting is now essential and, in some cases, long overdue. Before this can be done it is necessary to define the problems of existing systems and the aims of any proposed system.

As requests to the laboratory increase there comes a point where individual validation of each report becomes impossible, manual filing of results becomes inadequate, and an increasing percentage of reports is lost to the clinician and does not get into the patients' notes. Computers may be used to solve these problems but they must not be allowed to dictate the pattern of work and thus create their own organizational difficulties.

Data processing in haematology is primarily a question of filing and retrieval. Machines, such as $\mathcal{N}$ the Coulter S automatic blood counter (Coulter N Electronics, Dunstable), are available which print N digital results directly onto a report - a different situation from that in chemical pathology where the main aim of an automated data processing system $\stackrel{\varrho}{\subset}$

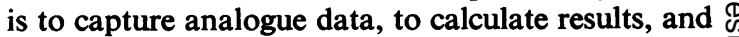
to produce a report. Moreover the worklists, accession numbers, and batch processing appropriate to a chemical pathology laboratory would introducy an unnecessary rigidity in the normal haematologe workflow. The basic problems in the two disciplines are different and we believe that, in the development 
stage at least, they must be treated as separate and distinct entities.

The aim of a haematology data processing system should be to gather all the data produced by a department into a single file from which it can be retrieved at will. This system will obviously be most efficient if the transmission of data to the file involves a minimum of transcription. Where automated apparatus (in most cases the Coulter $\mathbf{S}$ cell counter) is involved data capture should be automatic. The data in the file should be organized into two main parts: short-term, covering a matter of days, and long-term covering a complete patient history. The output from both should be in the form of a consolidated patient report but the time scale of retrieval should be a matter of seconds for shortterm retrieval while the latter could, except in emergencies, be much longer.

A quality control system that is capable of detecting changes in the accuracy and precision of the data is a necessary part of any laboratory activity. Because it is important to prevent invalid data being reported and entered into the file this should be a 'real-time' function which can interrupt the analytical process. Retrospective quality control is not an adequate alternative.

The basic requirements of a haematology data processing system can therefore be summarized as (1) the maintenance of a readily accessible cumulative patient file of all data; and (2) real-time quality control of automated apparatus.

Reporting results from the laboratory to the ward or clinic is an important consideration in the effectiveness of the service. A haematology data processing system can facilitate efficient reporting by producing collated reports for each ward or clinic but this is not a basic requirement.

\section{Strategy}

There are three possible ways in which a computerbased haematology system can be developed. First a completely independent processing and storage configuration could be designed. The high capital and running costs of such a system exclude its consideration in most cases. The second possibility is to develop a haematology system as part of a complete hospital system. High capital and running costs again make this impracticable for most laboratories. Moreover, a hospital computer system is generally introduced to solve financial and administrative problems. When haematology is incorporated in such a system the particular needs of the laboratory tend to be subordinated to those of the whole system.

The third, and we believe more promising, approach is to divide the computer functions into two. Data aquisition, on-line quality control, and short-term filing can be carried out on a small laboratory computer. This part of the system can be developed and implemented by the laboratory staff and can be made to suit the particular requirements of the laboratory. The capital costs of about $£ 10000$ are only a fraction of those involved in other approaches and additional staff are not required. Most regional hospital boards have a computer with the mass storage facilities necessary for maintaining long-term cumulative files. Data gathered by the in-lab computer can be transferred to the files of the central computer either directly over telephone lines or through a remote data entry terminal. This third approach is flexible and modular, is relatively cheap, and makes maximum use of the central resources of each hospital board. We believe that this is the optimal approach and one which is most generally applicable to haematology services throughout the country.

\section{The Cardiff Experiment}

A small computer has been used in the haematology laboratory at the University Hospital of Wales in conjunction with a large computer at the Welsh Hospital Board Computer Centre to test this third approach to data processing. The interrelationship of the laboratory and the computers is shown in figure 1 .

\section{PATIENT IDENTIFICATION}

Each patient is identified within the system by a hospital case record number. This number is linked to the patient's name, address, date of birth, and sex. The following details are filed when the patient is seen for the first time: a four-digit code identifying the hospital; a six-digit case record number; the first 12 characters of the surname; the first three characters of the first forename; the initial of the second forename; the first 50 characters for the patient's home address; the patient's date of birth; $M$ or $F$ to denote the patient's sex.

Patients who do not have a case record number, usually those referred directly to the department from their general practioner, are not included in the system at present. Their results are filed separately and manually. The feasibility of allocating a case record number to these patients is now being examined so that they can be included in the historical file. This will be of particular value in those cases referred to hospital at a later date.

THE LABORATORY SYSTEM

The Coulter $\mathbf{S}$ is interfaced directly with the labor- 


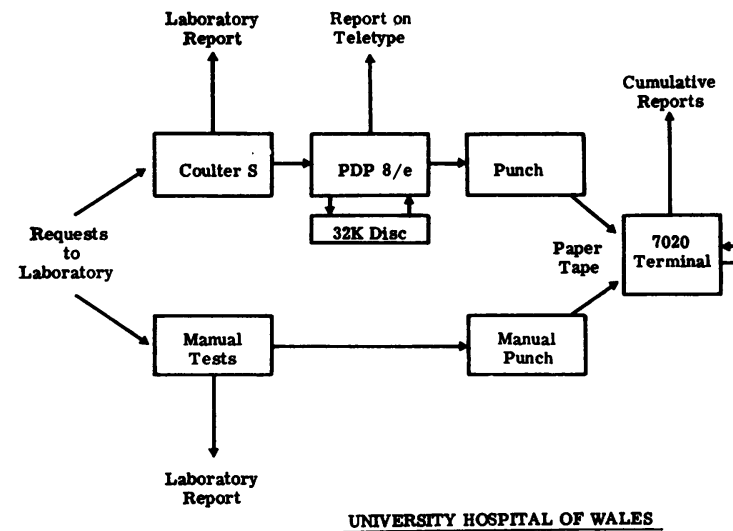

UNIVERSITY HOSPITAL OF WALES

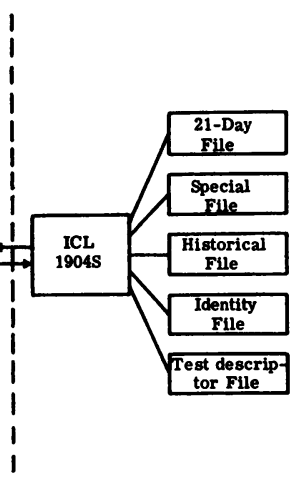

WELSH HOSPITAL BOARD COMPUTER CENTRE
Fig 1 Diagram showing the relationship of the laboratory to the central computer. atory computer which checks the physiological validity of all Coulter results and carries out realtime quality control as previously outlined (Cavill and Jacobs, 1973). The appropriate case record number is entered with each sample, merged with the Coulter results and stored on the disc as a single record. These records are punched to paper tape once a day and transmitted via a hospital terminal to the filing system on the Welsh Hospital Board computer. The laboratory computer also maintains an immediately accessible file of recent results to cover any lag in updating the main files. A diagram of the laboratory system is shown in figure 2. It is possible to interface additional equipment such as a second Coulter $\mathbf{S}$ or an automatic platelet counter with the laboratory computer.

\section{Equipment}

The laboratory computer is a PDP8/e (Digital Equipment Co Ltd, Reading, UK) with $8 \mathrm{~K}$ core

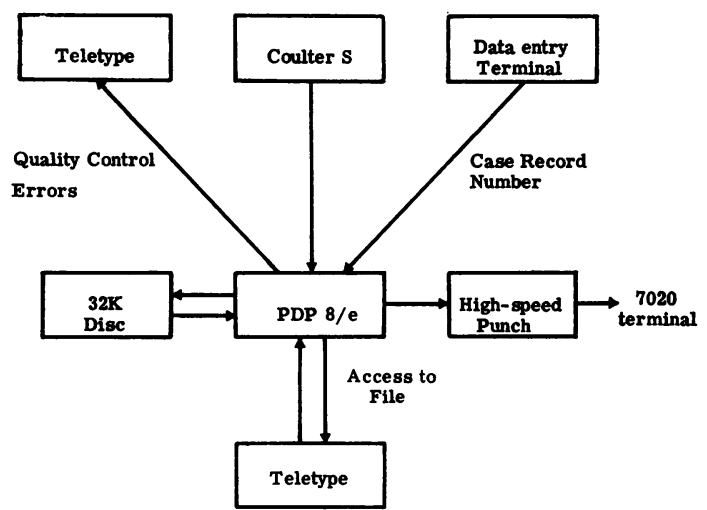

Fig 2 Diagram showing the relationship of the in-lab computer and its peripherals. of 12-bit words and a $32 \mathrm{~K}$ disc. There are also two teletypes, a high-speed paper tape reader/punch, $\frac{}{2}$ and a small data entry terminal with a keyboard $\vec{D}$ consisting of digits $0-9$, letters A-F, and a display of eight digits.

Results are input to the computer from the $\vec{\theta}$

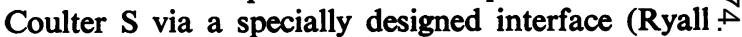
and Ricketts, 1974) as 12-bit, three-digit binary coded decimal numbers. Data are carried on 12 separate lines (S lines) and four other lines (Q lines) indicate which result is being transmitted. The Q1 line changes state between successive results and $\mathbb{\varnothing}$ this initiates an $80 \mathrm{~ms}$ delay before the result is read $\stackrel{2}{\vec{F}}$ into the processor.

\section{Programs and files}

The programs for this system were written and assembled in PAL-D (Digital, Disk Monitor? System). These operate under the computer's interrupt facility; appropriate input and output programs are entered after a short routine has $\frac{\circ}{3}$ determined which device caused the interrupt. The main programs occupy $4 \mathrm{~K}$ of core. The other $4 \mathrm{~K}$ ? is partly occupied by a standard software package $\frac{D}{0}$ for floating point decimal arithmetical operations used in quality control.

The results on the disc are held in two files. One contains the last 1024 records of patients' results. Each record occupies 11 words of storage and they $\omega$ are accumulated on the disc in the order in which they are input from the Coulter. When this file is full the oldest record is overwritten. The second file contains $\stackrel{\mathbb{D}}{\mathbb{D}}$ the results of the last 320 control samples. Each : record occupies 10 words of storage. The first two are the sample number and the date; the remaining $\underset{\mathbb{D}}{\vec{D}}$ eight contain the white cell count, red cell count, $\frac{?}{\mathbb{Q}}$

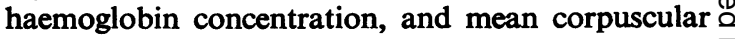
volume, and their cumulative sums. 
Operation

Operating the system in the laboratory simply involves entering the case record number at the data entry terminal as each sample is analysed. Other samples. such as controls, are identified by one of the characters A-F. At the start of each day the previous day's results are punched to paper tape and the current date entered. Results from the files can be retrieved at any time at the main teletype in the laboratory. Requests are made by typing '?' and then the case record number; all results filed with this case record number are then printed. A record can be recalled and deleted using the control character ' $D$ '. A second teletype at the Coulter S workstation is used to print messages giving warning of errors and failure of quality control.

\section{THE CENTRAL COMPUTER SYSTEM}

\section{Equipment}

The main archive for the whole system is based on the Welsh Hospital Board's ICL 1904 S (International Computers Ltd, London) computer with 96K of core, eight magnetic tape decks, and five EDS30 disc drives. Data are entered and retrieved via an ICL 7020 terminal with paper tape facilities and a line printer situated in the pathology area of the hospital. This provides an on-line link between the hospital and the central computer two miles away.

\section{Files}

The data collated by the main computer are organized into four main files: (1) patient identity file containing full identification particulars of all patients; (2) a file of recent results containing the last three weeks' data; (3) a historical file containing all results older than three weeks; (4) a special file of data on patients whose full records may need to be retrieved at short notice, eg, leukaemics, haemophiliacs.

\section{Operation}

All data are input as paper tape which consists of Coulter results produced by the laboratory computer and patient identification details and the results of other tests which are punched manually. Each result is qualified by a three-digit test code stamped onto the report form in the laboratory.
Cumulative reports may be produced from these files by specifying the patient's case record number. During the working day these reports can generally be produced within two hours of the request being made. For clinic appointments and scheduled admissions these reports are produced and appended to the notes on the day before the appointment.

\section{Conclusion}

By limiting our approach to the solution of the particular problems of the haematology department we have been able to develop a practical data processing system to serve the needs of the laboratory and the clinician. This has been achieved by separating the short- and long-term functions between a small on-line laboratory computer and a central mass storage computer. The result is a simple and inexpensive system which can be modulated to the particular circumstances of any haematology laboratory.

Over the past 18 months this system has been introduced into the laboratory and its development is continuing. The assistance of the computer in quality control has enabled us to institute a system of fast reporting of Coulter-generated results. The short-term file is being used increasingly for the rapid recall of past results. Cumulative reports from the long-term files are available both for outpatient clinics and scheduled hospital admissions and the clear presentation of all the available data on one or two sheets of paper has been an improvement on the case note record. This system has released a large part of the efforts of the clerical staff for more productive duties while in the laboratory the amount of time devoted to clerical duties has been substantially reduced. This, together with the interest derived from the active participation of the laboratory staff in the project, has contributed towards its ready acceptance. All of these developments have, we believe, improved the service that the laboratory has to offer and in those laboratories where the workload exceeds the capacity of manual data-processing methods, similar economical systems will need to be considered.

\section{References}

Cavill, I., and Jacobs, A. (1973). Quality control in haematology. Association of Clinical Pathologists Broadsheet 75.

Ryall, M., and Ricketts, C. (1974). In preparation. 